\title{
INCORPORATION OF Ag NANOPARTICLES INTO MICELLES. STABILITY STUDIES OF SELF-ORGANIZED NANOPARTICLES- MICELLES STRUCTURES
}

\author{
PRZEMYSLAW SIEJAK, KRZYSZTOF POLEWSKI
}

Department of Physics and Biophysics, Faculty of Food Sciences and Nutrition, Poznan University of Life Sciences, Poznan, Poland

Received February 17, 2016; accepted April 29, 2016; published online May 09, 2016

\begin{abstract}
In this paper we present the results of measured physical parameters of self-organized structures consisting of hydrophobic functionalized silver nanoparticles and amphiphilic molecules capable of micelles formation. Those systems may be considered as simple models for transfer of nanoparticles through the biological membrane. Three different surfactants were used: negatively charged sodium dodecyl sulphite, SDS, neutral Triton X-100 and positively charged tetredodecyltrimethyl ammonium bromide, TTABr. We have found that hydrophobic functionalized Ag nanoparticles are encapsulated in neutral Triton X-100 micelles with a diameter of $10 \mathrm{~nm}$ without significant change in the size of the micelles. The efficiency of encapsulation of Ag by SDS micelles is lower compared to Triron X-100 and no incorporation of Ag nanoparticles into TTABr occurs. Obtained results indicate that in aqueous environment ionic properties of molecules creating micelles and concentration ratios between components determine the efficiency and kinetics of two competitive processes association or aggregation of nanoparticles and encapsulation of Ag nanoparticles within micelles.
\end{abstract}

\section{INTRODUCTION}

Noble metal nanoparticles are considered for anticancer and antibacterial applications. It has been proved that nanoparticles are able to penetrate into selected tissues and bacterial cells, leading to their necrosis [Zhu \& Liao, 2015; Sosnowski, 2015; Dou Guo \& Ye, 2014; Chattopadhyay, Dash, Tripathy, Das, Mandal, Pramanik $\&$ Roy, 2014]. There is evidence of more efficient incorporation into cancer and bacterial cells with respect to unchanged tissues [Zhu \& Liao, 2015]. This phenomenon may be used for destroying cancer cells as well as for drug encapsulation and targeted delivery to selected cells [Dou et al., 2014]. Gold and silver nanoparticles of selected sizes are considered as the best reagents for above mentioned purposes, since they exhibit high bioavailability and no toxic influence of those particles on human has been found. However, recently much effort has been done to investigate other nanoparticles of different composition, sizes and shapes, with special interest on nanodiamonds. Nevertheless, the silver and gold nanoparticles are still in favor, even in common use. They are present in many popular preparations, including cosmetics, consumer goods, their packaging, as well as medical and paramedical products, and diet supplements. The understanding of the mechanism of incorporation and binding with selected parts of cells is essential for the prospective use of systems based on these various nanoparticles for diagnostic and therapeutic, as well as common purposes. The ability of nanoparticles to penetrate trough one or more layers of different biological membranes depends on many factors, like their size, shape, roughness, type of surface, electrical and ionic properties [Bothun, 2008]. All those factors determine the efficiency of nanoparticles incorporation into different parts (depth) of biological membrane, and thus the influence of nanoparticles on membrane components and membrane itself. It was shown that presence of nanoparticles in the membrane modify its flexibility and permeability [Bothun, 2008; Park, Oh, Mun \& Han, 2004, Park, Oh, Mun \& Han, 2006; Zhang, Sun, Song, Jiang, Dong \& Wang, 2006].

Moreover, the spectral properties of noble metal nanoparticles depend also on their shapes and sizes. The vivid colors of nanometric particles suspended in liquid are assigned to the plasma oscillations on the metallic surface, originating from coherent oscillations of free electrons gathered close to the surface with high density [Salamon, Macleod \& Tollin, 1997; Lakowicz, Malicka, Gryczynski \& Gryczynski, 2003a]. The resonance property of such complicated system determines the wavelength needed to excite surface plasmons. The absorption band of nanoparticles is interpreted as surface plasmon resonance peak and is believed to depend on the geometry of nanoparticles, especially on their 
homogeneity, size and shape [Lakowicz, Malicka, Gryczynski, Gryczynski \& Geddes, 2003b; Gryczynski, Malicka \& Gryczynski, 2004]. The excitation of surface plasmons, and distribution pathways of its energy can lead to significant changes in spectral properties of molecules located in close proximity to the metallic surface, leading to increase or decrease in its absorption or fluorescence. This phenomenon is widely used for monitoring of antibody binding kinetics, occurrence of specific chemical reactions and many other purposes [Aslan, Gryczynski, Malicka, Matveeva, Lakowicz \& Geddes, 2005; Spadavecchia, Manera, Quaranta, Siciliano, \& Rella, 2005; Soler, Mesa-Antunez, Estevez, Ruiz-Sanchez, Otte, Sepulveda, Collado, Mayorga, Torres, Perez-Inestrosa \& Lechuga, 2015].

Taking into account increasing interest in drugs and diet supplements containing colloidal metallic nanoparticles (especially gold and silver, in ex. Au-100, Ag-100 etc.), it is important to establish the influence of those metals on different parts of cell membrane and molecules present in the membrane. Moreover, the knowledge of precise location of nanoparticles in membrane in relation to another components of the layer and another molecules incorporated intentionally, and the results of metallic surface influence on the molecules can lead to development of different sort of bioindicators for application in food analysis [Spadavecchia et al., 2005], environmental monitoring and medical applications [Guo et al., 2014; Salamon et al., 1997; Lakowicz et al., 2003b; Aslan et al., 2005; He, Zhang, Feng, Song \& Zhao, 2011; Zhang, Mali, Aitken \& Geddes, 2013; Reddy, Yadav, Nagesha \& Karim, 2015]. Currently proposed system consisting of silver nanoparticles and surfactant molecules forming micelles can be considered as simple model system of monolayer vesicle trapping metallic nanoparticles. Taking into account the fact, that for many purposes the location of nanoparticles within the membrane, as well as the stability of produced system will be essential, we investigated the transfer of silver nanoparticles trough monolayer and stability of Ag-micelle system for different type of surfactant molecules at their premicellar and micellar concentrations.

\section{MATERIALS AND METHODS}

Decanethiol functionalized silver nanoparticles were purchased from Sigma Aldrich as a suspension in hexane. The silver content in purchased suspension was $0.1 \% \mathrm{w} / \mathrm{v}(0.1 \mathrm{mg} / \mathrm{ml})$. The size of nanoparticles was declared as $3 \mathrm{~nm}$ to $7 \mathrm{~nm}$ confirmed by DLS and TEM measurements. Micellar systems of sodium dodecyl sulphite (SDS) and tetradodecyltrimethyl ammonium bromide (TTABr) were prepared by dissolving of proper amount of surfactant molecules in water, to create $0.1 \mathrm{M}$ solution. Double distilled deionized water was used as a solvent and as a reference. $1 \mathrm{M}$ Triton X-100 purchased from Sigma Aldrich was used directly for investigation. In order to obtain surfactant solutions of lower concentrations, initial solutions were diluted using double distilled deionized water. For preparation of Agsurfactant and Ag-micellar systems, proper volume silver suspension in hexane was rapidly injected into aqueous environments, to assure silver content of 0.01 $\mathrm{mg} / \mathrm{ml}$ and $0.1 \mathrm{mg} / \mathrm{ml}$ in final system. The process was finished with complete hexane evaporation under ambient conditions.

Absorption spectra were recorded with Ocean Optics SD 1000 spectrometer. The Avantes DH-2000 UV/VIS lamp was used as the source of light. Sizes of micelles and particles were estimated by Dynamic Light Scattering (DLS) method performed with the use of Malvern ZetaSizer Nano ZS90 (90 deg geometry was applied). The accuracy of size of created structures was assessed on the basis of size distribution histogram. It was estimated as $10 \%$ for micelles and up to $15 \%$ (Triton X-100 based) and 20\% (SDS based) for Agmicelles structures.

The nanoparticles have been introduced into aqueous solutions of surfactants with concentration $0.12 \mathrm{CMC}$, $0.62 \mathrm{CMC}$ and emulsions of surfactant concentration 1.3 CMC to $12.5 \mathrm{CMC}$ for SDS, and additionally $50 \mathrm{CMC}$ and $100 \mathrm{CMC}$ for Triton X-100.

\section{RESULTS AND DISCUSSION}

\section{Absorption spectra}

The absorption spectra of hydrophobic functionalized silver nanoparticles in hexane are shown in fig. 1.

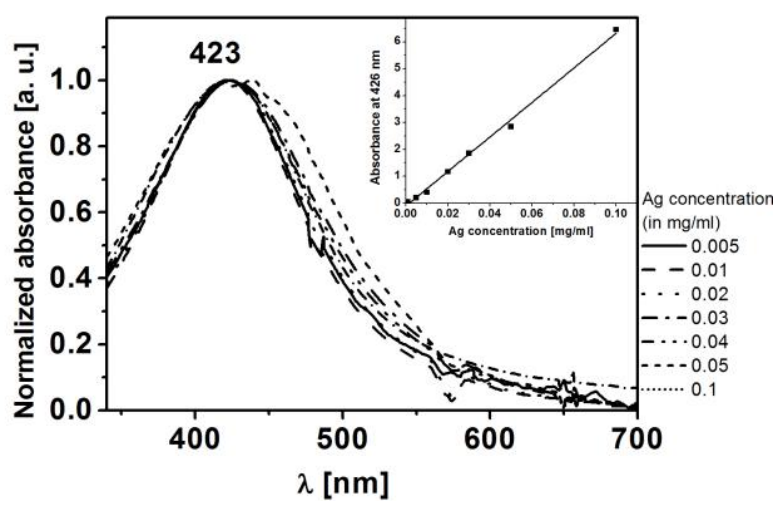

Fig.1. Normalized absorption spectra of Ag nanoparticles suspended in hexane for different $\mathrm{Ag}$ concentration; insert absorbance value changes as a function of Ag loading.

It presents normalized absorption spectra at different amount of nanoparticles (reported as w/V value). A wide 
absorption band is observed with maximum ranging from 423 to $429 \mathrm{~nm}$. With increasing nanoparticles concentration a slight shift of maximum (reported as surface plasmon resonance peak) towards the longer wavelengths was observed. The calculated FWHM were in the range of 124 to $147 \mathrm{~nm}$ (on average $134 \mathrm{~nm}$ ). The changes in FWHM are not determined by nanoparticles load in the suspension. The alteration of the value is rather stochastic, so occurrence of eventual additional effects, such as aggregation of nanoparticles or sedimentation can be excluded. The recorded spectra proved the homogeneity of used nanoparticles. Inset in fig.1 presents the absorbance of nanoparticles suspension versus its concentration (denoted as a w/V ratio). No fluorescence from silver nanoparticles suspension was observed. Based on results from fig.1 we may assume that nanoparticles can be treated as absorbing, non-scattering medium.

\section{Incorporation of nanoparticles into micelles}

In order to assess the incorporation feasibility of $\mathrm{Ag}$ nanoparticles into micellar vesicles and its time stability the absorption and dynamic light scattering measurements were carried out. The suspension of decanethiol functionalized silver nanoparticles in hexane had been injected into aqueous solution of micellar emulsion of SDS, Triton X-100 and TTABr and changes of absorption parameters as a function of time after injection was monitored. To establish the influence of nanoparticles on micelles formation a wide range of surfactant concentration was used. For two surfactants, SDS and Triton X-100, in presence of nanoparticles the changes in absorption spectra have been observed, fig. 2 . This phenomenon suggests that trapping of nanoparticles by micelles takes place. Neither considerable absorption changes was recorded for nanoparticles introduced into water, nor into TTABr micelles, as seen in fig.2. Therefore, TTABr emulsion was excluded from the further investigations.

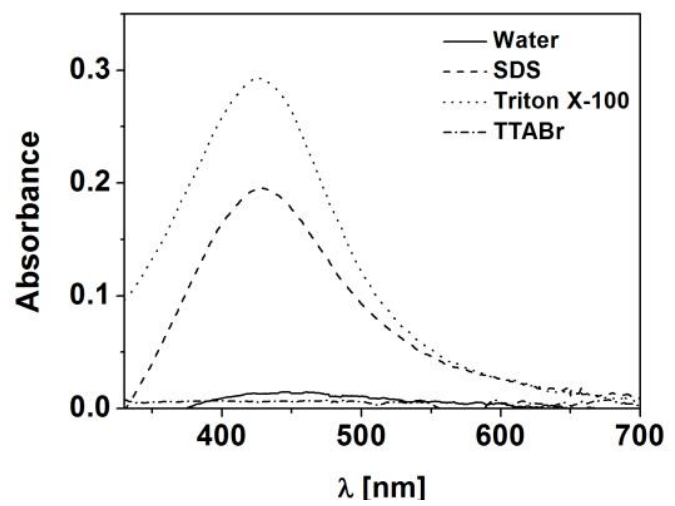

Fig.2. Absorption spectra of investigated systems for Ag loading $0.01 \mathrm{mg} / \mathrm{ml}$.
Nanoparticles introduced into water tend to aggregate and precipitate. The aggregation of silver nanoparticles in water has been manifested as formation of large visible clusters of silver. Presence of some aggregates and clusters of low diameter in water has been deduced based on minor absorption peaks with maximum at $460 \mathrm{~nm}-470 \mathrm{~nm}$. Systems formed as a result of $\mathrm{Ag}$ nanoparticles incorporated into SDS and Triton X-100 micelles were chosen for further analysis. On the basis of absorption values at maximum for $\mathrm{Ag}$ nanoparticles suspended in hexane and embedded in micelles the incorporation efficiency was roughly estimated. We assumed that the ratio of absorption value in micelles to the intensity in hexane is proportional to the number of nanoparticles trapped within micelles. The efficiency of Ag nanoparticles incorporation into micelles varies from $19 \%$ to $54 \%$ for SDS based systems and from $30 \%$ to $82 \%$ for Triton X-100 based systems, depending on surfactant concentration. The higher values are given for $0.01 \mathrm{mg} / \mathrm{ml}$ of $\mathrm{Ag}$ in suspension and the lower - for $0.1 \mathrm{mg} / \mathrm{ml}$. It reveals that a number of nanoparticles, or their clusters/aggregates locate inside of micelles whereas the rest precipitates. Directly after injection of Ag nanoparticles into aqueous solutions and emulsions, both phases separated rapidly and all nanoparticles tend to locate in non-aqueous medium. Limited and constantly decreasing volume of hexane causes oversaturation of suspension and precipitation of increasing number of nanoparticles from hexane to aqueous microemulsions. Ag nanoparticles coated with hydrophobic surfaces, tend to locate within micelles. The process of Ag transfer into micelle lasts until all hexane evaporates. This conclusion is supported by linear part of plot from fig.3, presenting absorbance value as a function of time after introducing nanoparticles in hexane into the aqueous medium. Visual inspection of each sample showed many precipitating nanoparticles, despite the presence of sufficient number of hydrophobic microvolumes.

The process of precipitation may be observed directly and it is manifested in changing absorbance values as a function of surfactant concentration. Moreover, as seen from fig.3, the raise of surfactant concentration, leading to increasing number of micelles, did not result in significant change of absorption. Increasing number of micelles by factor of 10 resulted in no more than $50 \%$ increase of maximum absorption, fig.3. From this same figure we may notice that after full hexane evaporation the increasing number of nanoparticles in the system resulted in proportional enhance in absorption value for low and high number of micelles. Thus, precipitation of nanoparticles or nanoparticle clusters is not a result of insufficient microenvironments providing stability of hydrophobic nanoparticles. From that, it can be concluded, that even at low concentration of surfactant, thus low amount of micelles, still there is sufficient 
number of vesicles to trap all the nanoparticles introduced into the medium. It is not clear why, despite the sufficient vesicles not all nanoparticles are located within the center of micelles, or are surrounded by micelle-like structures.

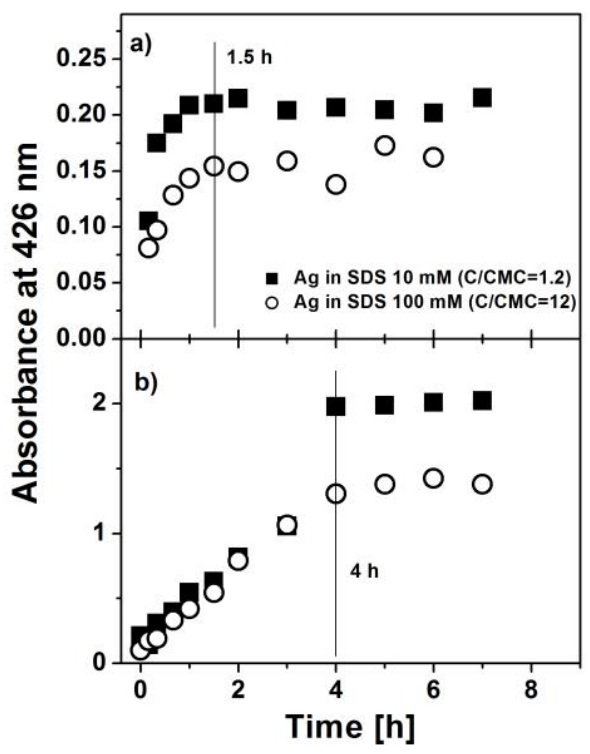

Fig.3. Time dependency of absorbance of silver nanoparticles at Ag loading $0.01 \mathrm{mg} / \mathrm{ml}$ (a) and $0.1 \mathrm{mg} / \mathrm{ml}$ (b) in SDS micelles during evaporation of hexane from the mixture - transition kinetics.

\section{Photophysical properties of nanoparticles located in micelles}

Comparing the spectra presented in fig. 1 and 2 one may reveal that those nanoparticles which locate in micelles do not change their optical properties relative to nanoparticles suspended in homogenous hydrophobic medium (hexane). The shapes of absorption band as well as the maximum wavelength of nanoparticles in micelles are similar to the spectra recorded for nanoparticles suspended in hexane. It can be assumed, that those parameters do not change as a result of transition trough the surfactant monolayer. The basic parameters of the plasmon bands of nanoparticles, especially, their FWHM are not sensitive to microenvironment, as long as the nanoparticles are present in the systems. The slight shift of absorption maximum of AgNPs located in vesicles relative to nanoparticles in hexane is seen in fig.1. This shift is not significant and do not exceed the changes observed for nanoparticles in hexane due to increasing number of AgNPs. The spectra recorded for nanoparticles in water differ significantly from all systems consisting of hydrophobic microenvironments. Very low absorption indicates negligible amount of nanoparticles suspended in pure water. Moreover, the absorption band is broad and the wavelength of maximum is considerably red shifted. The calculated FWHM of $136 \mathrm{~nm}$ for nanoparticles remaining in water does not differ significantly from the values obtained for micellar systems, $130 \mathrm{~nm}$ to $136 \mathrm{~nm}$. Surprisingly, those values are in agreement with results obtained for well defined, small nanoparticles in hexane, with diameter of single particle $3 \mathrm{~nm}$ to $7 \mathrm{~nm}$. The calculated FWHM of absorption band of $\mathrm{Ag}$ nanoparticles in hexane was calculated as $124 \mathrm{~nm}$ to $147 \mathrm{~nm}$. The results suggest that there is no change in size of single nanoparticles diameter wherever it is located in hydrophobic solvent or in aqueous medium.

\section{Geometry and size of Ag nanopaticles in water and in} micelles

The distributions of particle diameters for selected systems are presented in fig.4 whereas the mean particles diameter values calculated for the other systems are collected in Table 1.

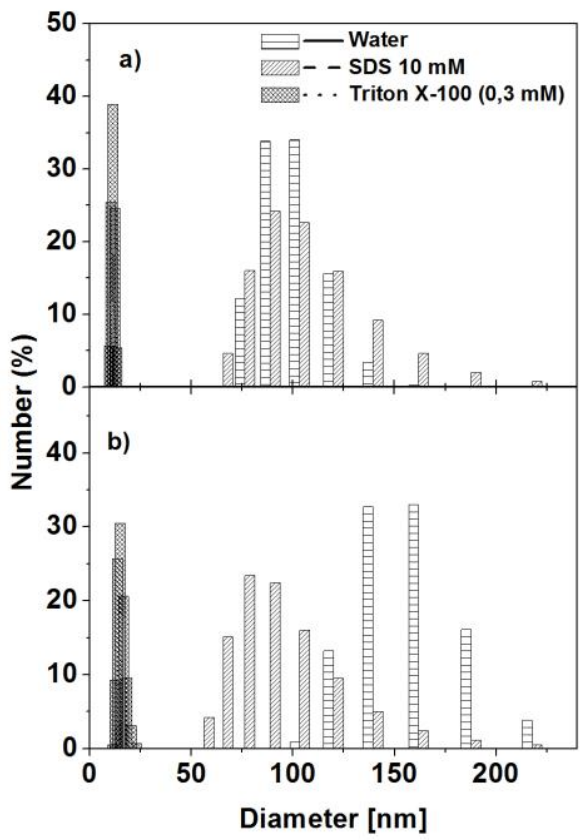

Fig.4. Size distribution of micelles containing $\mathrm{Ag}$ nanoparticles for Ag loading: $0.01 \mathrm{mg} / \mathrm{ml}$ (a) and $0.1 \mathrm{mg} / \mathrm{ml}$ (b) compared to nanoparticles in water. Surfactant concentrations are slightly above CMC (10 mM for SDS and $0.3 \mathrm{mM}$ for Triton X-100).

The results presented in fig. 4 show that the structures formed in water and SDS exhibit wide spread of size distribution with the mean size of $75 \mathrm{~nm}$ and higher, depending on solvent and concentration of nanoparticles. Contrary to the system in water and SDS, the sizes of nanoparticles located in Triton X-100 
micelles are well defined and ranging from $5.7 \mathrm{~nm}$ to $12.2 \mathrm{~nm}$. Those diameters can be assigned to AgNPs in hexane as well as in Triton X-100 micelles. The reported in many papers the size of SDS micelles is $2-4 \mathrm{~nm}$ [Varela, Macho \& Gonzales, 1995; Duplatre, Ferreira Marquez \& Graca Miguel, 1995] and for Triton X-100 micelles diameter is reported from 7.5 to $12 \mathrm{~nm}$ [Paradies, 1980; Dharaiya, Aswal \& Bahadur, 2015]. We estimated the size of SDS and Triton X-100 micelles as $3.34 \mathrm{~nm}$ and $12 \mathrm{~nm}$, respectively. The sizes were obtained for microemulsions of surfactants at concentration slightly above CMC - the calculated $\mathrm{C} / \mathrm{CMC}$ value was about 1.3. The micelle diameter decreases slightly as the surfactant concentrations increases. As shown in table 1, the dimensions of structures formed in SDS based systems are much larger than nanoparticles used and larger than reported diameter of vesicles. However, from absorption spectrum (fig.2) and changes of absorption during hexane evaporation (fig.3), we may notice that nanoparticles are distributed probably within the micelles of SDS or similar structures preventing some of nanoparticles from precipitation.

Based on obtained results, we may assume that either nanoparticles aggregate immediately after reaching aqueous environment and the large aggregates of nanoparticles are incorporated into micelles or formation of self-assembled aggregates of silver nanoparticles coated with micelles occurs. It might be also possible that initially the micelles are disintegrated and surfactant molecules form micelle-like large structure coating clusters of nanoparticles. The idea of decomposition of SDS micelles and forming of large clusters coating nanoparticles aggregates of different sizes can be supported by wide spread of sizes of particles composed of SDS molecules and silver nanoparticles (fig.4). The spread of the sizes of Ag-surfactant structures is even wider than for nanoparticles present in pure water. On the other hand, SDS micelles prevent the precipitation of nanoparticles, as shown in fig.2 and 4. Moreover, SDS prevents forming very large aggregates of silver nanoparticles at its high concentrations. From fig.4, it can be seen, that sizes of nanoparticles aggregates in water strongly depend on Ag concentration. They are much larger for systems containing high amount of nanoparticles $(0.1 \mathrm{mg} / \mathrm{ml})$ compared to lower concentrations $(0.01 \mathrm{mg} / \mathrm{ml})$, whereas particle sizes distribution for both concentrations of $\mathrm{Ag}$ in SDS microemulsions is similar.

From table 1 and fig.4 follows that aggregation of silver nanoparticles in aqueous medium does not take place for Triton X-100 micelles. This statement is supported by narrow distribution of particle sizes for aqueous suspensions containing both Triton X-100 micelles and silver nanoparticles. The distribution of particle sizes is slightly wider for higher silver nanoparticles content. However, negligible effect of presence of silver nanoparticles on micelle sizes indicates that $\mathrm{Ag}$ nanoparticles are transferred and located inside each micelle. Diameters of micelles and metallic nanoparticles are comparable, and negligible changes of vesicle sizes as a result of nanoparticles incorporation are observed. This effect suggests that one silver nanoparticle enters into one micelle, thus preventing $\mathrm{Ag}$ nanoparticles from aggregation and assurig stability of such structure.

Table 1. Changes in sizes of surfactant micelles as a result of $\mathrm{Ag}$ incorporation into vesicles for different concentration of surfactant molecules. $\mathrm{C}$ - concentration, CMC - critical micelle concentration, $\mathrm{d}_{0}$ - diameter of a micelle without Ag addition, $\mathrm{d}$ - diameter of a micelle with embedded $\mathrm{Ag}$.

\begin{tabular}{|c|c|c|c|c|c|c|c|c|c|c|c|c|}
\hline \multirow{3}{*}{$\mathrm{C} / \mathrm{CMC}$} & \multirow{3}{*}{$\begin{array}{c}\mathrm{C}_{\mathrm{SDS}} \\
{[\mathrm{mM}]}\end{array}$} & \multirow{3}{*}{$\begin{array}{c}\mathrm{C}_{\text {TRITON }} \\
{[\mathrm{mM}]}\end{array}$} & \multirow{3}{*}{$\begin{array}{c}\mathrm{d}_{0 \mathrm{SDS}} \\
{[\mathrm{nm}]}\end{array}$} & \multirow{3}{*}{$\begin{array}{c}\mathrm{d}_{0} \\
\text { TRITON } \\
{[\mathrm{nm}]}\end{array}$} & \multicolumn{4}{|c|}{$\mathrm{Ag}$ in SDS } & \multicolumn{4}{|c|}{ Ag in TRITON } \\
\hline & & & & & \multicolumn{2}{|c|}{$\mathrm{C}_{\mathrm{Ag}}=0.01 \mathrm{mg} / \mathrm{ml}$} & \multicolumn{2}{|c|}{$\mathrm{C}_{\mathrm{Ag}}=0.1 \mathrm{mg} / \mathrm{ml}$} & \multicolumn{2}{|c|}{$\mathrm{C}_{\mathrm{Ag}}=0.01 \mathrm{mg} / \mathrm{ml}$} & \multicolumn{2}{|c|}{$\mathrm{C}_{\mathrm{Ag}}=0.1 \mathrm{mg} / \mathrm{ml}$} \\
\hline & & & & & $\begin{array}{c}\mathrm{d} \\
{[\mathrm{nm}]}\end{array}$ & $\mathrm{d} / \mathrm{d}_{0}$ & $\begin{array}{c}\mathrm{d} \\
{[\mathrm{nm}]}\end{array}$ & $\mathrm{d} / \mathrm{d}_{0}$ & $\begin{array}{c}\mathrm{d} \\
{[\mathrm{nm}]}\end{array}$ & $\mathrm{d} / \mathrm{d}_{0}$ & $\begin{array}{c}\mathrm{d} \\
{[\mathrm{nm}]}\end{array}$ & $\mathrm{d} / \mathrm{d}_{0}$ \\
\hline 0.1 & 1 & 0.03 & & & 105.17 & & 110.10 & & 67.85 & & 103.40 & \\
\hline 0.6 & 5 & 0.15 & & & 111.87 & & 116.35 & & 15.57 & & 28.09 & \\
\hline 1.3 & 10 & 0.3 & 3.34 & 10.37 & 90.91 & 27.22 & 85.61 & 25.63 & 12.15 & 1.17 & 8.34 & 0.80 \\
\hline 2.4 & 20 & 0.58 & 1.62 & 7.73 & 55.33 & 34.20 & 90.31 & 55.82 & 9.55 & 1.24 & 9.79 & 1.27 \\
\hline 3.6 & 30 & 0.87 & 1.66 & 7.33 & 41.75 & 25.22 & 102.33 & 61.81 & 7.89 & 1.08 & 7.97 & 1.09 \\
\hline 6.3 & 50 & 1.5 & 1.56 & 5.08 & 56.83 & 36.55 & 73.41 & 47.21 & 8.86 & 1.74 & 7.98 & 1.57 \\
\hline 9.6 & 80 & 2.3 & 0.98 & 5.88 & 37.31 & 37.94 & 77.53 & 78.84 & 6.60 & 1.22 & 7.27 & 1.24 \\
\hline 12.5 & 100 & 3 & 0.72 & 6.06 & 46.83 & 64.69 & 42.96 & 59.35 & 6.36 & 1.05 & 7.5 & 1.24 \\
\hline 50.0 & & 12 & & 5.99 & & & & & 6.70 & 1.12 & 5.91 & 0.99 \\
\hline 100.0 & & 24 & & 6.06 & & & & & 5.68 & 0.94 & 6.08 & 1.00 \\
\hline
\end{tabular}


Time stability studies

The results from fig.3 show that absorption changes are related to hexane evaporation. Decreasing volume of hexane causes oversaturation of silver nanoparticles in hydrophobic medium and forces AgNPs to enter into aqueous phase. Moreover, form our measurements it follows that at insufficient hexane volume, nanoparticles are forced to enter to aqueous phase and occasionally locate in preferred microenvironment. Since inside of vesicles are only hydrophobic volumes, the nanoparticles tend to locate within micelles. To establish whether formed nanoparticles-surfactant or nanoparticles-micelle suspensions are stable, the absorption spectra were consequently recorded for 14 days after the time of full hexane evaporation for most of produced systems, including various surfactant concentration, thus various amount of micelles. All recorded spectra were compared to find differences inducing any physical changes of the each aqueous system with special interest on stability of metallic nanoparticles in the vesicles and eventual further precipitation of silver nanoparticles, their clusters or aggregates. As shown in fig. 2 and 5 no changes in absorption peak were found.

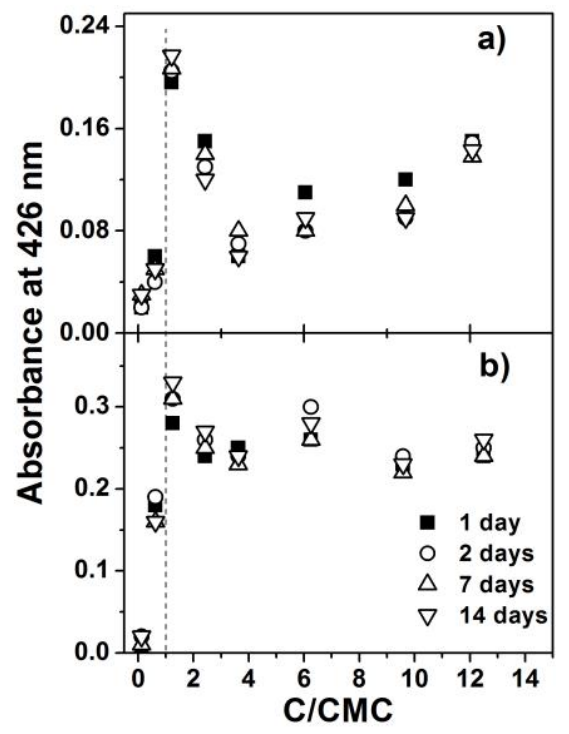

Fig.5. Peak absorption intensity of nanoparticles introduced into SDS (a) and Triton X-100 (b) solutions/micelles for $\mathrm{Ag}$ loading $0.01 \mathrm{mg} / \mathrm{ml}$. Dashed parallel line indicates $\mathrm{C} / \mathrm{CMC}=1$, meaning the lowest surfactant concentration for micelles formation.

Moreover, no changes in absorption band shape or FWHM was observed and the position of absorption maximum remained unchanged. Only small changes in absorbance value were observed. It can be concluded, that neither further aggregation nor precipitation of sliver nanoparticles takes place. Fig.5 presents the measured absorbance value at specified wavelength for selected Ag-micelle aqueous microemulsions covering the $\mathrm{C} / \mathrm{CMC}$ value from 0.03 to 12 . Based on the results from fig.5, it can be concluded, that additionally to previous statement, the changes of absorbance values are insignificant over the time for each created $\mathrm{Ag}$ nanoparticles in micelles suspensions in aqueous environment. This also reveals that once nanoparticles or their clusters enter vesicles or are trapped in other micelle-like systems, they remain stable and no more precipitation occurs. Similar conclusions concerning time stability and absorption parameters follows from the studies of systems containing high amount of $\mathrm{Ag}$ nanoparticles $(0.1 \mathrm{mg} / \mathrm{ml})$. The absorption intensity does not change significantly over the time of 14 days after preparation. The dependence of absorption intensity on $\mathrm{C} / \mathrm{CMC}$ for SDS based system containing $0.1 \mathrm{mg} / \mathrm{ml}$ of $\mathrm{Ag}$ is similar to the one presented in fig.5a. However for Triton X-100 micelles with high AgNPs load, there is no maximum of absorption intensity at $\mathrm{C} / \mathrm{CMC}$ close to unity, but plateau is observed. Moreover it is clearly seen that the efficiency of AgNPs incorporation into Triton X-100 micelles is nearly two times higher than for SDS. The result of $\mathrm{Ag}$ nanoprticles stability in inspected micelles for high $\mathrm{Ag}$ content, is presented in fig.6. Moreover calculated efficiency of $\mathrm{Ag}$ incorporation into Triton X-100 micelles for high amount of $\mathrm{Ag}$ at $\mathrm{C} / \mathrm{CMC}$ close to unity is much lower and equals $34 \%$.

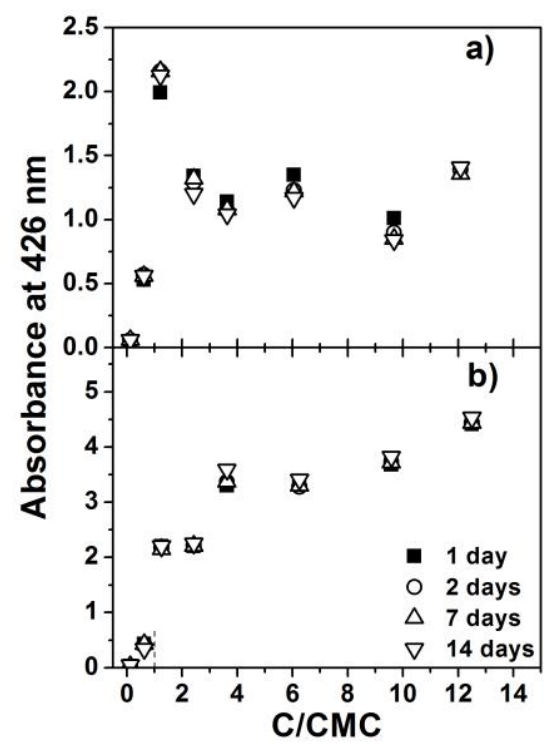

Fig.6. Peak absorption intensity of AgNPs introduced into SDS (a) and Triton X-100 (b) solutions/micelles for Ag loading $0.1 \mathrm{mg} / \mathrm{ml}$. 


\section{CONCLUSIONS}

The high time stability of produced Ag-surfactant micellar suspensions was observed in our studies. Minor oscillations of recorded absorbance value at different time may originate from light scattering by formed particles, which efficiency is rather stochastic. Taking into account all observations, it is important to stress out, that Ag nanoparticles can form stabile systems in micellar suspensions. Moreover, for most cases, there exist, specific concentration of surfactant molecules resulting in the highest efficiency of transition of nanoparticles trough monolayer. This preferable concentration of SDS is comparable to CMC value for investigated surfactants. In case of Triton X-100, that specific concentration of surfactant molecules can be clearly seen only for low amount of $\mathrm{Ag}$ nanoparticles $(0.01 \mathrm{mg} / \mathrm{ml})$ at $\mathrm{C} / \mathrm{CMC}$ close to 1 . Comparison of efficiency of $\mathrm{Ag}$ incorporation into micelles created by two examined surfactants, reveals, that Triton X-100 is much better for AgNPs encapsulation. Moreover it prevents Ag nanoparticles from aggregation before encapsulating. For SDS based systems, Ag nanoparticles form large clusters and are probably surrounded by SDS molecules creating structures of diameter up to 10 times larger than $\mathrm{Ag}$ nanoparticles introduced and 30 times higher than micelles.

\section{ACKNOWLEDGEMENTS}

The work has been supported by Poznan University of Life Sciences funds (no 507.782.57).

\section{REFERENCES}

Aslan K., Gryczynski I., Malicka J., Matveeva E., Lakowicz J.R. \& Geddes C.D.(2005). Metal-enhanced fluorescence: An emerging tool in biotechnology. Curr. Opin. Biotechnol., 16, 55-62.

Bothun G.D.(2008). Hydrophobic silver nanoparticles trapped in lipid bilayers: Size distribution, bilayer phase behavior, and optical properties. Journal of nanobiotechnology, 6, art. no 13 (only on-line journal).

Chattopadhyay S., Dash S.K, Tripathy S., Das B., Mandal D., Pramanik P. \& Roy S.(2014). Toxicity of cobalt oxide nanoparticles to normal cells; an in vitro and in vivo study. Chem. Biol. Interact., 226C, 58-71.

Dharaiya N., Aswal V.K. \& Bahadur P.(2015). Characterization of Triton X-100 and its oligomer (Tyloxapol) micelles vis-à-vis solubilization of bisphenol $\mathrm{A}$ by spectral and scattering techniques. Colloids Surfaces A Physicochem. Eng. Asp., 470, 230-239.

Dou, Q.Q., Guo H.C \& Ye E.(2014). Near-infrared upconversion nanoparticles for bio-applications. Mater. Sci. Eng. C. Mater. Biol., Appl. 45, 635-643.
Duplâtre G., Ferreira Marques M.F. Da Graça Miguel M.(1996). Size of sodium dodecyl sulfate micelles in aqueous solutions as studied by positron annihilation lifetime spectroscopy. J. Phys. Chem., 100, 16608-16612.

Gryczynski I., Malicka J., Gryczynski Z. \& Lakowicz J.R.(2004). Radiative decay engineering 4. Experimental studies of surface plasmon-coupled directional emission. Anal. Biochem., 324, 170-182.

Guo D., Zhang J., Huang Z., Jiang S. \& Gu N.(2014). Colloidal silver nanoparticles improve anti-leukemic drug efficacy via amplification of oxidative stress. Colloids Surf. B. Biointerfaces, 126C, 198-203.

He X., Zhang M., Feng J., Song M. \& Zhao X.(2011). New research progress of metallic silver enhanced fluorescence. Rare Met. Mater. Eng., 40, 559-564.

Lakowicz J.R., Malicka J, Gryczynski I. \& Gryczynski Z.(2003a). Directional surface plasmon-coupled emission: A new method for high sensitivity detection. Biochem. Biophys. Res. Commun., 307, 435-439.

Lakowicz J.R., Malicka J, Gryczynski I., Gryczynski Z. \& Geddes C.D.(2003b). Radiative decay engineering: the role of photonic mode density in biotechnology. J. Phys. D. Appl. Phys., 36, R240-R249.

Paradies H.H.(1980). Shape and size of a nonionic surfactant micelle. Triton X-100 in aqueous solution. J. Phys. Chem., 84, 599-607.

Park S.H., Oh S-G, Mun J-Y \& Han S-S.(2005). Effects of silver nanoparticles on the fluidity of bilayer in phospholipid liposome. Colloids Surf. B. Biointerfaces, 44, 117-122.

Park S.H., Oh S-G, Mun J-Y \& Han S-S.(2006). Loading of gold nanoparticles inside the DPPC bilayers of liposome and their effects on membrane fluidities. Colloids Surf. B. Biointerfaces, 48, 112-118.

Reddy B.P.K., Yadav H.K.S., Nagesha D.K., Raizaday A. \& Karim A.(2015). Polymeric Micelles as Novel Carriers for Poorly Soluble Drugs- Review. J. Nanosci. Nanotechnol., 15, 4009-4018.

Salamon Z., Macleod H., \& Tollin G.(1997). Surface plasmon resonance spectroscopy as a tool for investigating the biochemical and biophysical properties of membrane protein systems. II: Applications to biological systems. Biochim. Biophys. Acta, 1331, 131-152.

Soler M., Mesa-Antunez P., Estevez M-C., Ruiz-Sanchez A.J., Otte M.A., Sepulveda B., Collado D., Mayorga C., Torres M.J. Perez-Inestrosa E. \& Lechuga L.M.(2015). Highly sensitive dendrimer-based nanoplasmonic biosensor for drug allergy diagnosis. Biosens. Bioelectron., 66, 115-123.

Sosnowski T.R.(2015). Nanosized and Nanostructured Particles in Pulmonary Drug Delivery. J. Nanosci. Nanotechnol., 15, 3476-3487.

Spadavecchia J., Manera M.G., Quaranta F., Siciliano P. \& Rella R.(2005). Surface plamon resonance imaging of DNA based biosensors for potential applications in food analysis. Biosens. Bioelectron., 21, 894-900.

Varela A.S., Macho M.I.S. \& Gonzalez A.G.(1995). The size of sodium dodecyl sulfate micelles in the presence ofnalcohols as determined by fluorescence quenching measurements. Colloid Polym. Sci., 273, 876-880.

Zhang L., Sun X., Song Y., Jiang X, Dong S. \&, Wang E.(2006). Didodecyldimethylammonium bromide lipid bilayer-protected gold nanoparticles: synthesis, characterization, and self-assembly. Langmuir, 22, 2838-2843. 
Zhang Y, Mali B.L., Aitken C. \& Geddes C.D.(2013). Highly sensitive quantitation of human serum albumin in clinical samples for hypoproteinemia using metal-enhanced fluorescence. J. Fluoresc., 23, 187-192.

Zhu Y. \& Liao L.(2015). Applications of Nanoparticles for Anticancer Drug Delivery: A Review. J. Nanosci. Nanotechnol., 15, 4753-4773. 